Regards sur l'économie allemande

Bulletin économique du CIRAC

116-117| 2015

Varia

\title{
La réforme du dialogue social en France : les enseignements de l'expérience allemande
}

René Lasserre

\section{(2) OpenEdition}

\section{Journals}

Édition électronique

URL : http://journals.openedition.org/rea/4814

DOI : $10.4000 /$ rea.4814

ISSN : 1965-0787

Éditeur

CIRAC

Édition imprimée

Date de publication : 1 juillet 2015

Pagination : 29-42

ISSN : 1156-8992

\section{Référence électronique}

René Lasserre, «La réforme du dialogue social en France : les enseignements de l'expérience

allemande », Regards sur l'économie allemande [En ligne], 116-117 | juillet 2015, mis en ligne le 01 juillet 2017, consulté le 19 avril 2019. URL : http://journals.openedition.org/rea/4814 ; DOI : 10.4000/

rea.4814 


\section{La réforme du dialogue social en France : les enseignements de l'expérience allemande}

René Lasserre

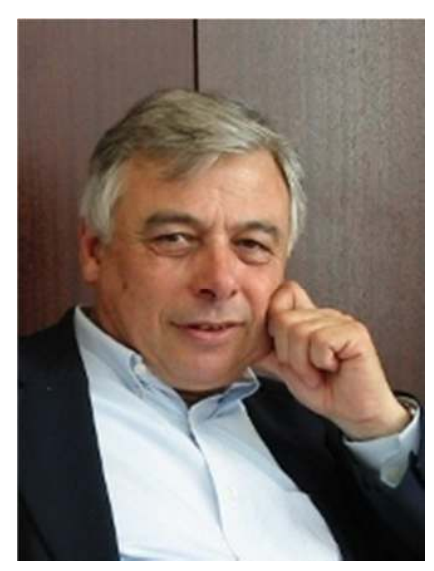

Prof. René Lasserre, Directeur du CIRAC
Cette contribution introductive à la réflexion actuellement en cours en France en vue d'une réforme du dialogue social a pour objet de mettre en évidence les différences systémiques les plus significatives qui séparent le système français et le système allemand de relations sociales. A partir de l'examen des principes organiques qui fondent l'efficacité du partenariat social allemand, elle vise à dégager des enseignements clés et des points incontournables pour une réforme du système français de relations professionnelles, avec l'objectif de mettre les partenaires sociaux en capacité d'engager et de promouvoir les "réformes structurelles » dont le pays a besoin.

Le propos s'inscrit dans la réflexion d'un groupe d'experts constitué à l'initiative de l'Institut Montaigne et ayant pour mission de formuler une série de propositions visant à rénover le dialogue social en France. Les points de vue que l'auteur développe ici, à partir d'une analyse fonctionnelle contrastive France-Allemagne, n'ont d'autre objet que d'apporter une contribution au débat et ne sauraient évidemment préjuger des propositions communes qui se dégageront des travaux du groupe de réflexion auquel il participe.

\section{Un système de régulation sociale français qui tourne à vide}

Le constat d'une déficience du dialogue social n'est pas nouveau au regard des difficultés chroniques qu'éprouvent en France les organisations syndicales et patronales à concilier leurs positions tant pour aménager des conditions de rémunération, d'emploi et de travail que pour assurer une protection sociale qui préservent la compétitivité des entreprises face à la concurrence mondiale. Sous l'effet de la crise, la situation s'est encore aggravée : les marges du partage se sont amenuisées pour devenir négatives et faire désormais place sinon à la réduction, du moins à une redéfinition des acquis. Dans ce contexte, le système de régulation sociale français, caractérisé par l'émiettement, la faible représentativité et, par voie de conséquence, l'autorité et la responsabilité réduites des organisations tant syndicales que patronales, a montré l'ampleur de ses déficiences fonctionnelles, déficiences très largement auto-entretenues, à la fois par la conjonction des idéologies tenaces et des corporatismes catégoriels étroits.

Ces déficiences sont celles d'un modèle qui privilégie une répartition conflictuelle de la création de valeur au gré des rapports de force, au détriment d'une gestion anticipative, concertée et responsable de l'appareil de production, en d'autres termes, d'un «pacte social de productivité » fondé sur l'équité, la promotion de l'investissement matériel et humain et la gestion collective de l'innovation. Pour s'en être largement remis à l'Etat pour gérer ces « externalités », voire en lui ayant abandonné de longue date le soin d'arbitrer en leur lieu et 
Un dialogue social qui se réduit à la protection des acquis place la répartition de la valeur ajoutée (notamment via le SMIC et la modulation des prélèvements sociaux), les partenaires sociaux se montrent avant tout soucieux, de part et d'autre, de la conservation des acquis. Et au besoin, lorsque ces acquis ne sont plus finançables, ils finissent par s'accorder, sous le couvert de la gestion tripartite de quelques régimes de prévoyance, sur une mutualisation publique de la dépense.

Dans le contexte régressif de la crise, ce repli défensif compromet un dialogue social constructif à travers lequel les partenaires sociaux pourraient, par des concessions réciproques, se redonner des marges de manœuvre et générer des gains de productivité tangibles. A privilégier les états-majors et faute de redonner la parole aux acteurs de terrain dans les branches et les entreprises, le modèle français de gestion professionnelle tripartite semble désormais à bout de souffle, pour ne pas dire englué dans une crise de gouvernance et de légitimité profonde.

C'est ce qu'illustre en particulier l'évolution récente de la négociation interprofessionnelle qui, après avoir été érigée par la loi Larcher en 2007 comme un préalable obligatoire à l'initiative régulatrice du législateur, a tenté de faire, depuis 2008-2009, de la promotion du dialogue social un enjeu central de modernisation sociale. Sept ans après, et alors que la crise appelait des ajustements tangibles à différents niveaux de régulation, les résultats des différentes phases de négociation sont en fin de compte assez maigres. En dépit des encouragements de la puissance publique, les partenaires sociaux n'ont pas repris l'initiative et ne sont tout au plus parvenus à s'entendre que sur des aménagements mineurs. Au final, les négociations se sont finalement soldées en janvier 2015 par un échec sur une question fonctionnelle centrale : celle de la mise en place, sous la forme du conseil d'entreprise élu, d'une seule et unique instance de représentation des salariés dans l'entreprise.

La réforme du dialogue social devrait au final intervenir sous la forme d'une loi dont le projet qui vient d'être présenté le 22 avril frise le ridicule puisqu'il propose, en guise de représentation des salariés dans les PME de moins de 50 salariés la désignation de délégués mobiles se réunissant périodiquement au siège du Conseil régional! La stratégie d'une réforme organique du dialogue social par la voie d'une négociation tripartite, supposant l'accord formel d'une part assez large des acteurs concernés, débouche ainsi sur une impasse, se heurtant de part et d'autre au veto d'organisations avant tout soucieuses de préserver leurs intérêts catégoriels ou leurs positions acquises.

Ce constat affligeant, auquel conduit finalement un cycle de trente ans de modernisation sociale engagé en 1982 avec les lois Auroux, justifie qu'une réflexion et un débat publics soient désormais engagés pour formuler, dans une perspective novatrice plus audacieuse que celle de la loi Larcher, un ensemble cohérent de propositions. En partant d'un diagnostic critique de l'expérience hexagonale, et tout en s'inspirant des principes de gouvernance sociale qui ont fait leurs preuves chez nombre de nos voisins européens, ces propositions devraient contribuer à lever les blocages d'un système de régulation qui ont progressivement étouffé la compétitivité de l'économie française et continuent d'en hypothéquer le redressement.

Contraste avec la capacité adaptative du système allemand
Dans cette perspective, le système de concertation sociale allemand constitue, tant par la cohérence de ses principes organiques que par l'efficacité de ses modes de régulation, une référence incontournable par la capacité adaptative dont il a fait preuve au fil des décennies, et plus encore dans la période de crise récente, dans la gestion concertée du changement. Une référence dont la portée reste cependant relative, à la fois parce que les conditions socio-institutionnelles qui, en Allemagne, président de longue date à la régulation sociale sont loin d'être immédiatement transposables dans l'Hexagone, et que, par ailleurs, les modes de cette régulation ne répondent que partiellement aux particularités du contexte économique et social français. 


\section{Les principes organiques \\ du système allemand de relations professionnelles et leur mise en œuvre dans le dialogue social}

L'expérience historique du système allemand de relations sociales est suffisamment riche et instructive pour que l'on s'y arrête. Ce dernier repose sur des principes que l'on peut qualifier d'organiques dans la mesure où ils sont profondément établis et reconnus par tous les acteurs, à la fois dans la pratique et dans l'esprit. Ils sont le fruit d'une expérience historique marquée par l'affrontement puis la synthèse entre deux tendances contradictoires, celle de l'étatisme social incarné initialement par la doctrine bismarckienne de l'Etat-providence et celle de la conquête autonome de droits sociaux portée par un mouvement social vigoureux au sein duquel l'approche réformiste l'a progressivement emporté sur la stratégie révolutionnaire de la lutte des classes. Après le dirigisme social de Weimar, imposé par des contraintes économiques difficiles, et qui vide progressivement un syndicalisme puissant mais divisé de sa raison d'être et fait le lit d'un nazisme destructeur de toute autonomie sociale, la refondation de 19481949 marque le retour au marché, et à son corollaire social, la liberté de négociation. Celle-ci apparaît alors à ce point régénératrice que l'on s'attachera à la garantir au rang de droit fondamental dans la Constitution de 1949, au même rang que les autres libertés, y compris celle du droit de propriété. Parallèlement, et conjointement à son caractère d'Etat de droit, à sa forme d'Etat fédéral, le nouvel Etat allemand se voit reconfirmer sa fonction d'Etat social (Sozialstaat), garant de la protection sociale.

Puisant aux racines d'une culture et d'une expérience sociales très riches, et sur cette base initiale de principes constitutionnels entièrement rénovée, le système social allemand se voit reconstruit sur trois piliers institutionnels.

L'Etat social, garant et responsable de la protection sociale, exerce à travers la loi fédérale ses prérogatives de tutelle publique sur les différents régimes légaux (obligatoires) de protection sociale, à la gestion desquels sont associées paritairement, à différents niveaux, les organisations professionnelles de salariés et d'employeurs. On notera d'emblée que cette prérogative régulatrice de l'Etat continue de s'exercer pleinement aujourd'hui. La gestation des réformes Schröder l'a illustré avec éclat : après avoir vainement tenté d'impliquer les partenaires sociaux dans la réforme d'un Etat-providence devenu économiquement insoutenable, le gouvernement fédéral a finalement décidé d'assumer seul la responsabilité politique de la refonte des régimes de protection sociale engagée en 2003 dans le cadre de l'Agenda 2010. Au-delà de son coût politique initial élevé - puisqu'elle a coûté le pouvoir au Chancelier Schröder -, cette réforme de l'Etat social a été résolument poursuivie par les deux majorités différentes suivantes et finalement largement couronnée de succès.

II convient toutefois de souligner que la réforme des régimes de protection sociale a été engagée avec retard, sous la pression d'une montée inquiétante du chômage, et postérieurement à celle du dialogue social menée conjointement et délibérément par les partenaires sociaux dans leur champ de responsabilité au tournant des années 2000 pour flexibiliser les rémunérations et les conditions d'emploi dans les grandes branches de l'économie allemande.

La négociation contractuelle des partenaires sociaux constitue en effet le second pilier du système social allemand. Elle est assurée de manière pleinement autonome et exclusive par les organisations professionnelles syndicales et patronales de branche, lesquelles ont souveraineté entière de négociation dans leurs branches respectives (les organisations confédérales ne peuvent négocier que par délégation des organisations de branche sur des sujets limités, étroitement circonscrits). La négociation concerne ainsi l'ensemble des conditions de rémunération (salaires, avantages sociaux), d'emploi et de travail (qualifications, formation, flexibilité) spécifiques à chaque branche, au niveau national ou
Liberté de négociation et protection sociale sont inscrites dans la Constitution

Trois piliers institutionnels

1. L'Etat social est garant et responsable de la protection sociale

2. La négociation contractuelle des partenaires sociaux assure la régulation de la vie au travail 
3. La législation du travail assure un ordre public social intangible régional. Chaque branche a ainsi ses normes sociales spécifiques (ex. : pas de réglementation uniforme du temps de travail) en fonction de ses conditions économiques et sociales propres, mais aussi de données régionales. Les normes conventionnelles s'imposent de fait, au besoin par procédure d'extension, à toutes les entreprises de la branche. Elles doivent satisfaire aux normes légales minimales de protection. La négociation de branche est à ce point structurante, spécifique et différenciée en fonction de la branche, qu'elle rend la négociation interprofessionnelle superflue et sans objet. Au-dessus de la norme conventionnelle n'existe que la norme légale du droit du travail commun, ce que l'on désigne en France sous le terme d'ordre public social, mais qui en Allemagne, du fait de l'étendue du champ de négociation, laisse à l'intervention du législateur un champ nettement plus restreint.

L'ordre public social constitue ainsi le troisième pilier du système. L'autonomie de négociation de la branche, de même que les règles qui président à la gestion sociale de l'entreprise, s'exercent dans le cadre de cet ordre public social, lequel procède de la législation du travail, essentiellement de droit fédéral (uniforme). Ce cadrage législatif se compose de droits individuels constituant des garanties-plancher et de droits collectifs offrant un cadre procédural stable et organisé à l'intérieur duquel s'exerce l'initiative normative des partenaires sociaux :

- la législation sur la protection individuelle des salariés assure de longue date à ces derniers des garanties sociales minimales (hygiène, sécurité, etc.) ou des droits spécifiques par catégories (femmes, apprentis...) ; la législation encadre également le contrat de travail et le licenciement, ainsi que les différentes formes de travail différencié ;

- le droit de négociation est encadré par la loi sur les conventions collectives (Tarifvertragsgesetz, 1949), laquelle se limite à définir pour l'essentiel (en 4 pages !) les parties soumises à négociation, la portée d'application et l'effet des conventions, ainsi que les modalités de leur extension. A ce texte est venu s'ajouter récemment, en août 2014, non sans objections de principe du point de vue du respect de l'autonomie contractuelle garantie par la Loi fondamentale, la loi sur la fixation d'un salaire minimum légal.

II est à noter que tous les autres aspects de la négociation, notamment son volet procédural, c'est-à-dire le contenu et la durée de validité des conventions, les modalités de leur conclusion et de leur dénonciation, ainsi que les procédures de conciliation, relèvent de l'autonomie pleine et entière de négociation évoquée précédemment. Ils sont définis par un accord-cadre de médiation de droit privé signé entre les partenaires sociaux de branche eux-mêmes, hors de toute intervention publique. Quant aux procédures de recours au conflit (grève vs lock-out), elles relèvent de dispositions statutaires propres aux organisations signataires. Les seules références sont d'ordre jurisprudentiel et relèvent en dernière instance du Tribunal fédéral du travail de Kassel.

L'exercice de l'autonomie contractuelle (i.e. liberté pleine et entière de négocier en dehors de toute intervention des pouvoirs publics), instaurée et garantie à l'article 9 de la Loi fondamentale de 1949, s'est révélé depuis lors l'atout essentiel de l'efficacité et de l'adaptabilité de la négociation, plus encore dans les périodes de crise où elle a facilité les ajustements structurels que dans les périodes fastes où elle moulinait inconsidérément des acquis sociaux difficilement réversibles. Cela s'est illustré au moment des chocs pétroliers, où elle a permis à l'industrie allemande de reconstituer rapidement et de consolider ses positions internationales. Ce fut également le cas vingt ans plus tard, dans la seconde moitié des années 1990, dans le contexte difficile d'une compétitivité mise à mal par les charges énormes de l'unification et une concurrence internationale exacerbée par la globalisation.

C'est à ce moment que la responsabilité économique et sociale conjointe des partenaires sociaux de branche a commencé à s'exercer pleinement au béné- 
fice de la compétitivité et de l'emploi à travers une politique contractuelle innovante et de plus en plus différenciée. Au-delà de la modération salariale, celle-ci intégra rapidement l'ensemble des dimensions du facteur travail, notamment la flexibilité du temps de travail et des conditions d'emploi et de rémunération, avant que ne s'instaure, au début des années 2000, dans les grandes branches industrielles exposées à la concurrence internationale, la pratique fréquente et autorégulée de la négociation dérogatoire en vue de préserver l'emploi et les sites de production domestiques. Ce qui eut par ailleurs pour effet de décentraliser la négociation et de renforcer considérablement le dialogue économique et le management concerté au niveau des entreprises.

On le voit : la négociation de branche en Allemagne, menée sous la responsabilité directe et exclusive des partenaires sociaux eux-mêmes, est le niveau de prédilection et l'instrument clé du partage primaire de la valeur ajoutée dégagée dans chacun des grands secteurs de l'économie marchande. En cela, elle est le processus de régulation autonome par lequel les acteurs sociaux d'un même secteur opèrent, par une négociation économique souveraine et responsable, un partage des gains de productivité et de la valeur ajoutée qui concilie le volume d'emploi et le niveau de rémunération, ainsi que le niveau de rentabilité et la capacité d'investissement des entreprises de la branche. Les "rounds tarifaires " de printemps auxquels donnent lieu les négociations récurrentes de branches sont autant de « conclaves » où se construisent, en dehors de l'Etat, les pactes de compétitivité de l'économie allemande.

En dépit, mais aussi en raison de la prégnance des accords de branche, la concertation et la négociation d'entreprise n'ont cessé de s'enrichir pour adapter, infléchir et compléter l'application des normes conventionnelle de la branche et ce, par le fait même que la législation offre un cadre stable et opératoire à la concertation sociale entre la direction de l'entreprise et le personnel.

La loi sur la représentation dans l'entreprise (initiée dès 1920, refondée en 1949 sous le terme de Betriebsverfassungsgesetz, réamendée à deux reprises depuis lors) constitue le droit commun. Elle ne prévoit qu'une seule et unique instance de représentation des salariés sous la forme d'un conseil d'entreprise élu (Betriebsrat) à partir de 5 salariés (avec un seul représentant jusqu'à 20 , trois de 21 à 50 , cinq de 51 à 150 salariés, 11 de 600 à 1000 , puis 4 par millier supplémentaire). Le Betriebsrat détient et exerce l'ensemble des prérogatives de représentation des intérêts sociaux du personnel de l'entreprise. Les représentants syndicaux peuvent y siéger avec voix consultative. Les domaines d'attribution du Conseil sont très étendus et concernent aussi bien les questions sociales, la formation, les questions de gestion collective et individuelle du personnel et des apprentis, que les questions économiques régulières et les changements importants.

L'étendue des attributions est modulée selon les domaines en fonction des degrés de souveraineté des parties prenantes : elles vont d'un droit de co-décision de portée très substantielle, qui s'étend à toutes les questions sociales, aux mesures collectives relatives à la formation et à la gestion du personnel. Ceci implique que, dans ces domaines clés de la gestion sociale, aucune décision du chef d'entreprise ne peut être prise sans l'approbation et l'accord formels du Conseil d'entreprise, et qu'en cas de désaccord, la décision doit faire l'objet d'une procédure de conciliation interne obligatoire devant une commission paritaire restreinte entre le $\mathrm{CE}$ et la direction, laquelle conduit généralement à un compromis. Le recours à la juridiction du travail prévu en cas d'échec est possible mais peu fréquent. Loin d'être une source de blocage, cette disposition contribue à une culture de dialogue responsable et constructif, dans l'intérêt conjoint des parties prenantes de l'entreprise. De plus, les décisions conjointes prises d'un commun accord, y compris à l'issue d'une procédure de conciliation, peuvent faire l'objet d'un accord d'entreprise (Betriebsvereinbarung) formalisé, immédiatement applicable.
Le droit de représentation et la co-décision des salariés contribuent à la cohésion de l'entreprise 
Dans le domaine des mesures individuelles de personnel, notamment en matière de licenciement, le Conseil est saisi et dispose d'un pouvoir de recours et d'objection éventuel sur le bien-fondé et la régularité de la décision en principe souveraine du chef d'entreprise. L'avis du CE est transmis, parallèlement au motif invoqué par l'employeur, à l'appréciation du juge qui statue en dernière instance sur la régularité du licenciement.

Dans le domaine économique, le conseil dispose d'un droit d'information régulier et étendu de la part de la direction de l'entreprise. II dispose en outre d'un pouvoir consultatif significatif par le biais d'une commission économique obligatoire dans les entreprises de plus de 100 salariés. Mais dès lors que, dans une entreprise de plus de 20 salariés, intervient une décision économique impliquant des conséquences sociales directes sur l'emploi et l'affectation d'une partie du personnel, le chef d'entreprise doit établir un plan social auquel le conseil d'entreprise doit donner impérativement son accord. A défaut, une commission de conciliation, statuant au besoin en présence du président de l'office régional de l'emploi, établit un plan social définitif prévoyant une indemnisation appropriée.

On le voit : par sa légitimité et l'étendue de ses attributions, le Betriebsrat allemand dispose d'une capacité d'influence incontournable dans la gestion du facteur travail. II est par là même un acteur co-responsable du changement social et de la valorisation des ressources humaines dans l'entreprise allemande. II est en outre le bras articulé qui, au niveau de l'entreprise, met en œuvre et prolonge les normes négociées de la convention de branche, voire adapte et met en œuvre ces dernières dans le cadre de la négociation dérogatoire. Loin d'être un frein au changement, il est de fait, conjointement avec la direction de l'entreprise, et dans le respect des intérêts des salariés, le promoteur de la compétitivité sociale de l'entreprise allemande, aussi bien dans les grandes entreprises, et plus souvent encore dans les PME.

La cogestion dans les grandes entreprises (Mitbestimmung) est un régime particulier qui s'applique aux grandes entreprises, instaurée initialement en 1951 dans le secteur charbon-acier et généralisée en 1976 dans l'ensemble des sociétés anonymes de plus de 2000 salariés. Elle se traduit par une représentation paritaire et avec voix délibérative des représentants élus des salariés aux côtés de ceux des actionnaires dans les conseils de surveillance. En cela, elle constitue un régime d'exception puisqu'elle assure une participation des salariés aux choix stratégiques des sociétés, mais elle ne concerne que quelque 500 sociétés. Elle présente de ce fait une portée d'ampleur limitée. A mentionner cependant la représentation minoritaire des salariés à un tiers des sièges dans les conseils de surveillance de toutes les sociétés de plus de 500 salariés qui est plus largement répandue. Mais au regard des spécificités du modèle rhénan de gouvernance, l'un et l'autre dispositif présentent, pour la modernisation du système français, une valeur heuristique réduite.

\section{La problématique de la modernisation du dialogue social français}

L’omniprésence codificatrice de l'Etat et la prééminence de la loi dans la régulation sociale..

...réduisent la portée régulatrice de la négociation de branche...
En République fédérale, au pays du libéralisme organisé, le poids et la fonction de la loi sont, comme on l'a vu précédemment, loin de jouer un rôle négligeable, mais ont une fonction radicalement différente de celle qu'elle tient dans l'Etat jacobin dont l'un des premiers actes fondateurs a été, avec la loi Le Chapelier de 1791, de supprimer les corps de représentation sociale intermédiaires. Alors qu'en Allemagne, la loi constitue le cadre organisateur à l'intérieur duquel se développe la régulation sociale autonome des partenaires sociaux, la loi constitue en France, à tous les niveaux, non seulement le cadre structurant, mais l'instrument privilégié de la régulation sociale elle-même. La séparation systémique entre cadre et processus, inhérente à l'ordo-libéralisme et qui prévaut en Allemagne dans le domaine économique et social, est inconnue en France.

L'omniprésence du législateur (consacrée par la loi fondatrice du 11 février 1950) et la prééminence de la loi qui en découle réduisent structurellement le champ de la négociation des partenaires sociaux, et ce aux deux niveaux fonc- 
tionnels où ils peuvent naturellement l'exercer pour réguler leurs intérêts conjoints. D'abord à celui de la branche professionnelle, où il est pertinent et souhaitable, en fonction des conditions techniques économiques et sociales communes à l'activité de production dans un espace géographique donné, de définir des conditions homogènes de qualification, de rémunération et de travail pour les salariés du secteur considéré. A celui de l'entreprise ensuite, en fonction des conditions spécifiques de son activité propre. La prééminence du recours à la loi conduit à réguler, selon des normes uniformément applicables à l'ensemble des secteurs d'activité et des entreprises et à l'échelle de tout le territoire, des variables aussi importantes que la durée hebdomadaire du travail ou le salaire minimal. Cela a pour effet de vider la négociation collective de branche de l'essentiel de sa fonction et de son contenu, et partant, de dessaisir les acteurs de leur capacité à réguler ses enjeux économiques et sociaux principaux. La négociation se trouve ainsi réduite à un rôle complémentaire sur des domaines annexes, lequel est par ailleurs lui-même fortement corseté par des clauses obligatoires définies par la loi pour en assurer la portée juridique effective dans le cadre de la procédure de sa possible extension de validité à l'ensemble de la branche par voie réglementaire. Avec ce double verrouillage, la négociation de branche en France est condamnée à végéter. On est loin de la fonction structurante que joue encore très largement, et en toute autonomie de ces acteurs, la négociation de branche dans la plupart des grands secteurs de l'économie allemande.

Après avoir été éclipsée pendant trois décennies, et bien que rendue finalement obligatoire par la loi Auroux du 13 novembre 1982, mais de la compétence exclusive des délégués syndicaux, la négociation d'entreprise a mis longtemps à prendre pied dans le système français de relations professionnelles. Elle ne s'est progressivement acquis une importance et une fréquence significatives qu'à travers les possibilités ouvertes par les lois de 1996 sur l'aménagement du temps de travail et celle du 13 janvier 2000 sur la semaine de 35 heures. Depuis lors, les entreprises de plus de 50 salariés se sont vu offrir, par les lois du 4 mai 2004 et 20 août 2008, dans leur partie relative au dialogue social, et en fonction des contraintes d'adaptation qu'elles rencontrent, la possibilité de conclure des accords dérogatoires à des garanties légales ou conventionnelles existantes. Ces dispositions n'ont toutefois donné lieu qu'à des avancées très timides (Dares, 2008), car là encore, la négociation d'entreprise demeure étroitement enserrée dans une hiérarchie de normes et un carcan de procédures qui en limitent l'application dans le tissu fragile des PME et en rendent la mise en œuvre très contraignante au regard de l'étroitesse des libertés consenties. On mesure ici encore la distance qui sépare cette pratique conditionnelle et étroitement surveillée de la négociation dans l'entreprise française et celle, autonome, normale et récurrente de la Betriebsvereinbarung allemande qui complète à la fois les normes de la convention de branche et consigne régulièrement les résultats de la co-décision entre le conseil et la direction de l'établissement.

Face à la faiblesse chronique de la négociation classique et à l'étroitesse persistante de son champ de régulation, l'évolution la plus significative enregistrée dans le paysage français des relations professionnelles des vingt-cinq dernières années est incontestablement la place grandissante de la négociation interprofessionnelle menée au sommet par les pouvoirs publics, en l'occurrence le ministre du Travail, et les directions nationales des organisations patronales et syndicales. A travers cette importance de la négociation centralisée au sommet, on mesure toute l'antinomie de logique qui sépare la régulation sociale française de celle pratiquée en Allemagne, où elle n'a d'existence que ponctuelle, sous la forme très épisodique de consultations d'intérêt public au cours desquelles pouvoirs publics et partenaires sociaux expriment leur volonté commune d'agir conjointement face à de grands défis (Pactes pour l'emploi ou pour la formation professionnelle).
... et étouffent la négociation d'entreprise

Importance croissante de la négociation interprofessionnelle centralisée 
Figure de proue du dialogue social à la française, la négociation interprofessionnelle donne lieu, du fait de la pluralité des organisations tant patronales que syndicales, à des discussions formalisées dans lesquelles les acteurs se montrent souvent plus soucieux de faire valoir leurs intérêts divergents que de parvenir à des compromis opératoires. Amorcée dans les années cinquante avec la mise en place de régimes de retraites complémentaires, poursuivie dans les années soixante avec la mise en place de l'assurance chômage, puis dans les années soixante-dix avec la promotion d'un droit individuel et d'un financement socialisé de la formation continue, la mensualisation des salaires ou l'extension des congés payés, cette forme de négociation a fait ses preuves. Les accords nationaux interprofessionnels (ANI) sont devenus progressivement l'instrument privilégié de promotion conjointe, entre Etat et partenaires sociaux, d'avantages ou de droits sociaux uniformes bénéficiant à l'ensemble des salariés, quelle que soit la dynamique économique et sociale de leur secteur d'activité. Bref, le vecteur d'une politique contractuelle mutualisée, désignée sous le terme générique et consensuel de paritarisme, pur produit des années de croissance. Initiée et pilotée par l'Etat, avalisée par les employeurs dans les limites des gains de productivité jugés redistribuables au gré de la conjoncture et « engrangée " par les organisations syndicales en fonction de leurs cahiers de revendications respectives, cette machinerie du dialogue social était celle que l'on désigne depuis lors, non sans nostalgie, comme celle qui répartissait « le grain à moudre ".

Même si, à la suite des chocs pétroliers puis de la globalisation progressive des activités, les conditions macro-économiques et les différenciations sectorielles ont très sensiblement réduit son efficacité, la négociation interprofessionnelle a conservé une très large légitimité institutionnelle, de sorte qu'elle est restée une voie de concertation privilégiée pour aborder de nouveaux enjeux. Ce fut tout d'abord le cas avec la réduction et l'aménagement du temps de travail au cours des années 1990 où elle joua de plus en plus nettement le rôle de terrain exploratoire à l'élaboration de la loi. Avant de se restreindre progressivement, depuis le début des années 2000 , sous la contrainte d'une croissance ralentie, à des chantiers de modernisation qualitative, principalement dans le champ des modalités du dialogue social, avec des thèmes tels que ceux de la négociation dérogatoire ou de l'aménagement des structures de représentation.

La loi Larcher : un point d'orgue sans suite

Dans ce processus de glissement des enjeux, la négociation interprofessionnelle change toutefois radicalement de fonction : elle devient de plus en plus l'antichambre préparatoire de la législation ordonnatrice, un lieu de consultation préalable avec les responsables sociaux, où sont censés s'élaborer des compromis opératoires avec la participation active des partenaires sociaux. C'est cette évolution qu'a cherché à promouvoir, jusqu'en la codifiant, la loi Larcher du 30 janvier 2007, dite de modernisation du dialogue social. Celle-ci prévoit d'associer systématiquement les partenaires sociaux, sous la forme d'une consultation débouchant sur une négociation préalable, à tout projet gouvernemental de réforme des relations du travail, de l'emploi et de la formation professionnelle. Assez largement saluée tant par les acteurs professionnels que les forces politiques, cette loi a plus fait l'objet d'un succès d'estime qu'elle n'a été couronnée d'efficacité. Elle relevait davantage d'une logique procédurale formaliste qu'elle n'a donné lieu, dans le contexte de la crise, à une stratégie ordonnée de modernisation axée sur des enjeux précis, et de ce fait même s'est heurtée au scepticisme des acteurs économiques.

Procéder à des consultations formelles effectives avant toute réforme conséquente de la régulation du travail n'est pas en soi problématique tant que les changements attendus peuvent recueillir de la part des acteurs un consensus assez large pour pouvoir s'appliquer effectivement sur le terrain et servir ainsi de base à des lois instaurant un ordre public social efficace et légitime. L'exercice a cependant montré au cours des dix dernières années que ce n'est en règle générale que faiblement le cas, tant les états-majors sociaux répugnent à 
engager leur responsabilité sur des changements significatifs dans les règles du jeu et s'attachent à préserver des privilèges catégoriels générant des rigidités supplémentaires. Au final, les accords ne sont généralement signés que par une partie des organisations dont l'audience globale n'atteint que rarement une majorité de salariés, ce qui, loin de l'en empêcher, incite plutôt le législateur à transcrire ces accords imparfaits sous forme de loi pour en assurer l'application. C'est ainsi que, sur la base de consensus fragiles et composites, s'élabore une loi générale de portée réduite qui n'est finalement que peu appliquée.

Dans ce cas de figure désormais habituel, la négociation interprofessionnelle présente à l'expérience beaucoup d'ambivalence et exerce une fonction doublement négative. D'une part parce qu'elle n'est bien souvent qu'une négociation en trompe-l'œil, qui débouche sur une législation a minima n'apportant en fin de compte que des changements à la marge. D'autre part, parce qu'étant tributaire du consentement initial des acteurs institutionnels, elle s'engage à structure constante et ne modifie en rien l'édifice d'ensemble des règles du jeu existant. Elle consolide ainsi l'étroitesse du champ de la négociation dans les branches et dans les entreprises, là où le dialogue social, s'il disposait d'une autonomie suffisante pour s'exprimer, pourrait permettre aux acteurs de terrain d'agir de concert sur les vrais leviers de la compétitivité.

Plus inquiétant est le fait que cette forme de négociation centralisée, qui tend à subordonner l'essentiel de l'initiative régulatrice au plus petit commun dénominateur d'organisations sociales peu représentatives et porteuses d'intérêts disparates, est davantage un facteur d'immobilisme qu'elle ne permet d'innovation réelle dans le dialogue social. Le recours préférentiel des gouvernants des majorités successives à cette forme de négociation-consultation préalable témoigne en fait de leur pusillanimité chronique à faire face et à imposer des arbitrages à un concert d'acteurs sociaux désunis s'érigeant en porte-paroles d'intérêts particuliers, voire de groupes-vétos. En cela, le rituel de la concertation au sommet procède d'une forme caractérisée de renonciation du législateur à promouvoir, de lui-même et au nom de la responsabilité politique qui est la sienne, une législation du travail conforme à l'intérêt général. De ce point de vue, la négociation interprofessionnelle semble, dans son évolution récente, loin de répondre aux vertus qu'on a coutume de lui attribuer. Sous couvert d'une recherche de consensus de bon aloi, cette pratique de la loi pré-négociée s'appuie sur une légitimité professionnelle de plus en plus étroite et porte en germe une dérive régressive inquiétante vers le corporatisme.

C'est dire, avec on ne peut plus de netteté, que la promesse de campagne émise en 2012 par le candidat-président François Hollande de " constitutionnaliser " cette procédure de dialogue social définie dans la loi Larcher doit être non seulement considérée avec la plus grande circonspection juridique et politique, mais purement et simplement écartée. Cela reviendrait en quelque sorte à l'ériger en processus organique de contrôle professionnel obligatoire préalable de l'activité du législateur qui risque tout simplement de conduire à la paralysie. II conviendrait au contraire, pour l'affranchir de l'immobilisme politique à laquelle elle préside, de sortir du champ de la négociation interprofessionnnelle toute initiative réglementaire relevant de l'ordre public social, a fortiori lorsqu'elle concerne la composition et les attributions des instances de représentation sociale. Cette prérogative relève foncièrement des pouvoirs du législateur et ne saurait faire l'objet d'une quelconque forme de visa ou de veto contractuel préalable, voire de mandat impératif de la part des acteurs professionnels qui en sont directement partie prenante.

Une acception bien comprise du principe de subsidiarité n'implique nullement un tel dévoiement sous la forme d'une consultation institutionnalisée et contraignante des partenaires sociaux dans tous domaines et au plus haut niveau. II semble au contraire urgent et nécessaire de mettre fin au mélange des genres et à la confusion des pouvoirs en ramenant la négociation interprofessionnelle
Le renforcement de la négociation interprofessionnelle : un palliatif en trompe-l'œil

De l'immobilisme au corporatisme

Replacer la négociation professionnelle dans son cadre initial 
Régulation sociale et compétitivité

Deux modes de régulation très contrastés

Remettre les partenaires sociaux au centre du dispositif de régulation dans son domaine originel de compétence qui relève à proprement parler du paritarisme. Celui-ci recouvre la gestion tripartite des régimes complémentaires de protection sociale (retraites complémentaires, assurance chômage...) ou la mutualisation de l'effort de formation professionnelle mis en place sur la base d'un financement conjoint des partenaires sociaux. II reste qu'en ce domaine également, une clarification s'impose : participation à la gestion des régimes de prévoyance implique également co-responsabilité financière et acceptation des impératifs de soutenabilité qu'il appartient à la puissance publique d'assurer en dernier ressort.

L'évolution de l'architecture du système français de régulation sociale fait ainsi apparaître des constantes, voire certaines dérives dirigistes et corporatistes qui continuent de le distinguer fortement et même, par maints aspects, de façon accentuée, de celles de son homologue allemand. Cela ne laisse pas d'interpeller quant à l'incidence de cette évolution sur la performance économique et sociale respective des deux pays, alors que dans la même période ils ont été l'un et l'autre confrontés, dans le contexte européen et mondial, à des facteurs de changement très largement communs.

Cette question met cependant en jeu de multiples variables structurelles qui dépassent largement le cadre de cette analyse, laquelle est tout au plus centrée sur la capacité respective des deux systèmes à opérer une répartition optimale de la productivité entre le facteur travail et le facteur capital aux différents niveaux de régulation et à promouvoir ainsi un cadre de régulation sociale favorable à une compétitivité économique durable.

Nous sommes en effet en présence de deux modes de régulation très contrastés. Le système allemand privilégie les niveaux de régulation les plus proches de la création de valeur que sont la branche et l'entreprise pour un face-à-face de négociation directe sur les principales composantes de la rémunération (salaire et avantages conventionnels, prestations complémentaires d'entreprise) et sur les conditions d'emploi (classifications, formation, conditions de promotion, travail différencié...) et de travail (temps de travail, flexibilité, congés, etc.). Ces différentes dimensions sont régulées de manière très variable d'un secteur et aussi d'une région à l'autre, mais dans tous les cas, elles sont directement tributaires de la valeur ajoutée dégagée dans la profession et l'entreprise.

Du côté français, le système de rémunération salariale et de détermination des conditions de travail donne au contraire une très large place à une régulation uniforme pour ainsi dire socialisée, puisqu'elle intervient sur la base de dispositions d'ordre public définies par la loi et applicables à toutes les entreprises quel que soit leur secteur d'activité, leur implantation géographique ou leur structure, notamment dans leur intensité en main-d'œuvre. Cela vaut en particulier pour le SMIC dont les effets pervers sur la masse et la structure salariales sont manifestes par le gonflement qu'il provoque dans le premier tiers de la pyramide des emplois et le tassement qu'il induit sur l'ensemble de l'échelle des rémunérations (Cette et al., 2012). De même pour la durée hebdomadaire légale du travail fixée uniformément à 35 heures, et aussi pour nombre de composantes du contrat de travail relevant de dispositions légales. Sous couvert d'équité sociale, l'ordre public social, lorsqu'il s'applique aux éléments de régulation du coût ou des conditions d'emploi de la main-d'œuvre, provoque de graves distorsions globalement préjudiciables à la compétitivité des entreprises et à l'emploi.

Face à l'autonomie dont disposent leurs homologues allemands, les acteurs professionnels français sont privés de leviers essentiels pour pouvoir assurer sciemment et efficacement la compétitivité de leur activité, et ce aussi bien au niveau de l'entreprise qu'à celui des branches. L'interventionnisme législatif et réglementaire envahissant et instable de la puissance publique en matière sociale prive en effet les acteurs économiques et sociaux d'une part importante de leur souveraineté contractuelle. La négociation de branche voit ainsi sa fonction réduite pour l'essentiel à la fixation de normes minimales que l'entreprise com- 
plète ex post en fonction de ses marges. Inversant ainsi les rôles, l'intervention publique prive en même temps le syndicalisme et les organisations professionnelles de leur raison d'être et plus gravement encore de leur compétence régulatrice. La revitalisation du dialogue social et, par là même, celle de l'influence et de la crédibilité des partenaires sociaux français, passe par un désarmement graduel, mais effectif, de la tutelle réglementaire et régulatrice qu'exerce l'Etat sur la sphère du travail.

\section{Enseignements de l'expérience et des pratiques allemandes pour un aggiornamento social français}

L'expérience et la pratique allemandes du dialogue social nous fournissent ainsi, par comparaison, des éléments de réflexion précieux, tant du point de vue des principes organiques qui président à la gouvernance du système qu'à celui des niveaux et des modalités de la régulation.

Si l'on considère le premier niveau, celui de la gouvernance globale des deux systèmes, il apparaît d'emblée, à la lumière de la comparaison France-Allemagne, que l'existence d'un soubassement constitutionnel explicite et cohérent, tel qu'il a été mis en place au rang des droits fondamentaux par la Loi fondamentale à son article 9 , est un élément essentiel pour assurer la légitimité, l'efficacité et la stabilité d'un système de régulation sociale.

En France, où pourtant dans le discours, le progrès social semble faire figure de raison d'Etat, il n'existe pas «d'ordre constitutionnel social » digne de ce nom. Le Préambule de 1946, fondé essentiellement sur les libertés individuelles du citoyen, mentionne en tête le droit de grève et en second lieu la représentation des intérêts sociaux du salarié dans l'entreprise, sans mentionner explicitement la négociation en tant que telle. Le Préambule énonce davantage des références historiquement datées, remontant aux conquêtes sociales du Front populaire, qu'il n'évoque des principes d'organisation et des modes de représentation des intérêts dans les relations du travail. Au regard de l'évolution des rapports sociaux en France depuis 70 ans, le temps semblerait peut être venu de donner une base constitutionnelle à la modernisation sociale, allant au-delà d'une déclaration des droits du travail (Badinter/Lyon-Caen, 2015).

Et, puisque dans le discours social, la perspective ne semble désormais plus exclue de constitutionnaliser le dialogue social, il semblerait opportun d'explorer cette voie. Non plus pour instaurer de simples procédures de consultation, du type de celle préconisée par la loi Larcher - ce qui apparaît incongru -, mais bien pour affirmer et faire prévaloir des principes organiques qui devraient enfin permettre de mettre, par la reconnaissance d'un droit à négociation autonome, les partenaires sociaux au centre du dispositif de régulation sociale.

Dans l'état actuel d'instabilité, d'immobilisme et de paralysie du paysage de régulation sociale français, sans parler de l'état de faiblesse des organisations syndicales et professionnelles, la remise au centre du jeu des acteurs sociaux aux différents niveaux où s'organise la vie de travail semble la meilleure voie possible pour redonner substance au dialogue social. Par la prééminence qui devrait être ainsi donnée au droit et au pouvoir de négocier, la norme conventionnelle se verrait reconnaître comme élément intangible de régulation des conditions de rémunération, d'emploi et de travail.

Pour être légitime au regard de la tradition politique et sociale française, cette autonomie de négociation devrait être assortie, en contrepartie, de la mention explicite de la capacité du législateur d'encadrer cette liberté de négocier par des dispositions d'ordre public social garantissant les droits sociaux dans des domaines de référence clairement circonscrits.

Sur cette base constitutionnelle, il conviendrait en particulier au législateur, en dehors du champ clairement défini d'exercice de ses prérogatives en matière
L'enjeu de la constitutionnalisation des relations sociales

Constitutionnaliser le droit des partenaires sociaux à la négociation autonome 
Revitalisation et renforcement de la négociation de branche

Le renouveau incertain de la négociation de branche de fixation d'un ordre public social minimal, de définir les principes généraux et les procédures de la négociation en indiquant notamment aux différents niveaux, principalement la branche et l'entreprise, les modalités de négociation et les partenaires habilités à négocier. La puissance publique garderait enfin ses prérogatives en matière d'extension de validité des conventions, ce qui, dans un contexte de pluralisme et de faiblesse des organisations, restera une prérogative importante. Resteront également à déterminer en droit, mais surtout en pratique, l'importance et les modalités respectives de la négociation dans les branches et dans l'entreprise. A ce niveau, les enseignements de l'expérience allemande seront sans aucun doute précieux, mais pas pour autant facilement transposables, tant le tissu économique des branches que l'organisation de l'entreprise sont différents de part et d'autre du Rhin.

La pratique allemande démontre le bien-fondé et l'efficacité d'une négociation de branche récurrente, dynamique et différenciée selon ses principaux objets : rémunérations, classifications, formation et qualifications, conditions d'emploi et de travail, lesquelles contribuent à définir de façon prévisible des conditions d'emploi homogènes et compatibles avec les enjeux économiques communs (compétitivité-prix, productivité, flexibilité, innovation) d'une même branche d'activité. Le renforcement de la négociation sectorielle et de sa récurrence demeure en France un objectif important au regard de la dispersion des branches, de l'étroitesse de leur périmètre, de leur hétérogénéité, sans parler de leurs conflits d'intérêts internes entre grandes et petites et moyennes entreprises, et plus encore entre grands donneurs d'ordre et sous-traitants.

Cette dispersion et cette hétérogénéité devraient pouvoir être progressivement, mais partiellement, surmontées à travers le relèvement progressif des critères de représentativité des organisations signataires requis pour la validation et l'extension des accords. L'incitation qu'une libération et une extension du champ de la négociation contractuelle sur des domaines clés des rémunérations, des conditions d'emploi et de travail apporterait à une intervention contractuelle devrait contribuer au regroupement progressif des branches professionnelles. Le processus risque cependant d'être assez long dans la mesure où la pratique et les compétences des branches se sont très sensiblement réduites au fil des années et que le maillage contractuel s'est beaucoup distendu avec l'hétérogénéité croissante du tissu entrepreneurial.

De plus, la structure sectorielle de l'économie française est très différente de celle de l'Allemagne où prédominent à la fois des branches industrielles puissantes, fortement structurées et intégrées, et où coexistent des liens de coopération étroits entre grands groupes, un Mittelstand composé d'entreprises intermédiaires puissantes et de PME dynamiques. Une structure de type analogue prévaut également dans les principales branches du secteur des services marchands. Dans cette configuration, la négociation salariale et sociale de branche est en quelque sorte consubstantielle de la performance de branche, et un facteur puissant d'égalisation des conditions de concurrence du fait de l'importance que tiennent les relations commerciales extérieures.

En France, les perspectives d'un renouveau de la négociation de branche apparaissent a priori beaucoup plus modestes du fait de la structure économique du pays. De ce côté-ci du Rhin, le tissu économique et entrepreneurial est beaucoup plus hétérogène et fragmenté. II est polarisé entre les grands groupes évoluant pour une large part à l'échelle multinationale, et un tissu éclaté de PME de taille modeste ou réduite, dont les caractéristiques et la structure de coûts sont également très différenciées selon qu'elles évoluent plutôt dans les secteurs classiques sur le marché intérieur ou régional, ou au contraire dans les secteurs technologiques selon une logique infra- et/ou multinationale de niche. Dans ce cas, la dynamique concurrentielle est beaucoup plus largement déterminée par la capacité d'innovation que par le coût salarial. Et en règle générale, la PME française ne tire que peu d'avantages d'une solidarité 
contractuelle très largement préemptée par l'emprise des prélèvements publics et doit l'essentiel de sa performance à sa capacité interne à mobiliser ses collaborateurs. Au final, et sur la base de ce paysage entrepreneurial hexagonal, il apparaît que c'est plutôt au niveau de l'entreprise qu'à celui de la branche que le déverrouillage de la négociation contractuelle semble susceptible de porter ses fruits et devait ouvrir de nouvelles dynamiques et des perspectives réelles de régénération. La refondation des conditions d'un dialogue social efficace dans l'entreprise n'en apparaît en fin de compte que plus essentielle.

La comparaison avec l'Allemagne fait apparaître l'inextricable confusion et la faiblesse dramatique du dispositif français. L'ordre du jour est à la réforme et prend pour l'instant, à travers les avant-projets actuellement présentés, des voies dont certaines méritent d'être sérieusement explorées et d'autres apparaissent d'emblée inconsistantes, pour ne pas dire absurdes. Relève de ce registre le projet d'une représentation spécifique des TPE menée à distance par des délégués régionaux itinérants...

La seule voie sérieuse qui mérite à mon sens d'être examinée en profondeur est celle d'un regroupement effectif des instances de représentation du personnel (IRP) autour d'une instance unique dont le principe n'est pas acquis, et dont les contours, la composition, et plus encore les attributions restent flous. La question la plus importante, qui est encore loin d'être tranchée, est cependant celle du choix de la logique de représentation entre la représentation par des membres élus par le personnel et celle assurée par des délégués désignés par les organisations syndicales.

L'expérience allemande montre la pertinence d'une répartition des modes de représentation par niveaux, la négociation de branche restant l'apanage exclusif de la représentation syndicale, celle de l'entreprise reposant prioritairement sur des membres élus par le personnel de l'entreprise, agissant en tant que tels et bénéficiant d'une légitimité effective auprès des salariés de l'entreprise, ce qui n'exclut nullement une appartenance syndicale personnelle pour environ les trois quarts d'entre eux.

Au vu du pluralisme syndical français, de ses corollaires que sont la plus faible représentativité et la moindre légitimité des organisations au plan de l'entreprise, on serait tenté de privilégier la formule allemande de la représentation élue, d'autant que l'efficacité de la représentation suppose l'existence d'un rapport de confiance solide avec l'employeur. Néanmoins, il convient, pour satisfaire à l'objectif éminemment souhaitable d'un renforcement de représentativité du syndicalisme français, de lui donner la possibilité d'agir et de démontrer sa crédibilité au contact direct des salariés, c'est-à-dire en premier lieu sur le terrain de l'entreprise. Ce qui me semble, en toute objectivité, devoir plaider pour l'instauration d'un conseil d'entreprise élu, une instance unique de représentation mixte, dans laquelle à partir d'un certain seuil, représentants élus et délégués syndicaux pourraient être représentés à parité et agir de concert face à leur employeur. Et ce avec de larges prérogatives, notamment en matière de négociation.

La question de l'ampleur des attributions et du degré de contrôle qui devraient leur être reconnus ne fait pour l'instant pas encore l'objet d'un réel débat, ce qui semble impliquer tacitement que l'on se satisfasse de pouvoirs consultatifs. La question de la co-décision selon les modalités allemandes de la conciliation interne, ou selon l'approche française de la négociation d'accords, reste ouverte. Tout indique, au regard de la tradition française où la coopération entre salariés et employeur ne s'engage pas a priori en pleine confiance (vertrauensvoll, dit la loi allemande), qu'une négociation périodique serait plus facilement praticable. On imagine mal qu'à un moment ou à un autre, cette question ne doive être abordée. L'essentiel n'en demeure pas moins qu'il convient, dans une négociation-maison qui se voit destinée à devenir la voie privilégiée de fixation autonome des conditions de rémunérations et de travail dans l'entreprise, de con-
L'incontournable et décisive réforme de la représentation des salariés dans l'entreprise

Instaurer un Conseil d'entreprise comme instance unique de représentation mixte 
cilier les deux modes de représentation reconnus par le droit français (élection/ désignation) en les réunissant dans une instance mixte de représentation unique légitimée de surcroît par le suffrage. Cela représenterait en outre l'avantage de légitimer les délégués syndicaux et de renforcer leur crédibilité. Une voie à explorer pour refonder le dialogue social sur le terrain. La synthèse d'approches françaises et allemandes mérite d'être examinée, voire expérimentée. La modernisation du dialogue social suppose aussi de promouvoir de nouveaux acteurs porteurs de nouvelles pratiques.

LA REFERENCE AU MODELE ALLEMAND POUR PENSER LA MODERNISATION des relations sociales françaises n'est pas un simple exercice d'école. Elle n'est pas sans intérêt, tant la communauté de pensée qui réunit nos deux pays en matière de droits sociaux est forte et présente des complémentarités insoupçonnées, même si elle s'articule dans nos deux pays selon des modalités d'action différentes en fonction de l'expérience et des circonstances.

Faisant abstraction de cette réalité, on se réfugiera derrière des préventions tenaces en affirmant que l'expérience allemande n'est pas transposable en raison d'une histoire sociale et d'une tradition culturelle différentes. Raison de plus : à l'heure où le modèle social français connaît une grave crise d'efficacité et de confiance, n'est-il pas fertile, et porteur d'avenir pour une Europe unie, de comprendre par quelle lumière de la pensée et de l'action nos voisins ont reconstruit solidement le leur au lendemain de l'expérience la plus tragique de leur histoire?

\section{Indications bibliographiques}

- AdAM G., BasiLIEN J.-P., Reconstruire le dialogue social, Rapport juin 2011, Institut Montaigne/ Entreprise et personnel, Paris, 2011

- Badinter R., Lyon-Caen A., Le travail et la Loi, Fayard, Paris, 2015

- Batelemy J., Cette G., Refondation du droit social : concilier protection des travailleurs et efficacité économique, La Documentation française, Paris, 2013

- Cette G., Chouard U., Verdugo G., "Les effets des hausses du SMIC sur le salaire moyen », in Economie et Statistiques, $\mathrm{n}^{\circ}$ 448-449, 2012

- Chertier D.-J., Pour une modernisation du dialogue social. Rapport au Premier ministre, 2006

- DARES, Evaluation de la Loi du 4 mai 2004 sur la négociation dérogatoire dans l'entreprise, par Merliaux O. et al., in Document d'études, n 140, août 2008

- FerRaci M., Guyot F., Dialogue social et performance économique, Presses de Sciences Po, Paris, 2015

- Lallement M., Sociologie des relations professionnelles, La Découverte, Paris, 2008

- LASSERRE R., «Partenariat social : la fin de l'exception allemande ? ", in Stark H., Weinachter M. (eds), L'Allemagne unifiée 20 ans après la chute du Mur, Presses universitaires du Septentrion, Valenciennes, 2009. 